Experimental and theoretical cross sections for positron scattering from the pentane isomers

L. Chiari' , A. Zecca, F. Blanco, G. García, and M. J. Brunger

Citation: J. Chem. Phys. 144, 084301 (2016); doi: 10.1063/1.4942472

View online: http://dx.doi.org/10.1063/1.4942472

View Table of Contents: http://aip.scitation.org/toc/jcp/144/8

Published by the American Institute of Physics

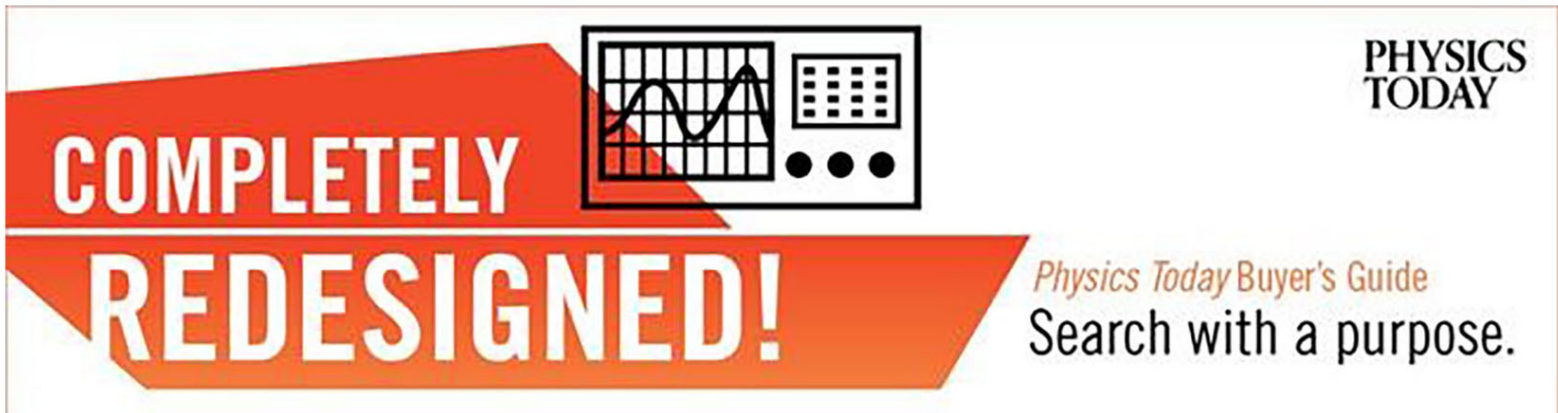




\title{
Experimental and theoretical cross sections for positron scattering from the pentane isomers
}

\author{
L. Chiari, ${ }^{1,2, a)}$ A. Zecca, ${ }^{3}$ F. Blanco, ${ }^{4}$ G. García, ${ }^{5}$ and M. J. Brunger ${ }^{2,6}$ \\ ${ }^{1}$ Department of Physics, Tokyo University of Science, 1-3 Kagurazaka, Shinjuku, Tokyo 162-8601, Japan \\ ${ }^{2}$ School of Chemical and Physical Sciences, Flinders University, GPO Box 2100, Adelaide, SA 5001, Australia \\ ${ }^{3}$ Department of Physics, University of Trento, Via Sommarive 14, Povo, Trento 38123, Italy \\ ${ }^{4}$ Departamento de Física Atomica, Molecular y Nuclear, Universidad Complutense de Madrid, \\ 28040 Madrid, Spain \\ ${ }^{5}$ Instituto de Física Fundamental, Consejo Superior de Investigationes Científicas (CSIC), Serrano 113-bis, \\ 28006 Madrid, Spain \\ ${ }^{6}$ Institute of Mathematical Sciences, University of Malaya, 50603 Kuala Lumpur, Malaysia
}

(Received 18 December 2015; accepted 8 February 2016; published online 24 February 2016)

\begin{abstract}
Isomerism is ubiquitous in chemistry, physics, and biology. In atomic and molecular physics, in particular, isomer effects are well known in electron-impact phenomena; however, very little is known for positron collisions. Here we report on a set of experimental and theoretical cross sections for low-energy positron scattering from the three structural isomers of pentane: normal-pentane, isopentane, and neopentane. Total cross sections for positron scattering from normal-pentane and isopentane were measured at the University of Trento at incident energies between 0.1 and $50 \mathrm{eV}$. Calculations of the total cross sections, integral cross sections for elastic scattering, positronium formation, and electronic excitations plus direct ionization, as well as elastic differential cross sections were computed for all three isomers between 1 and $1000 \mathrm{eV}$ using the independent atom model with screening corrected additivity rule. No definitive evidence of a significant isomer effect in positron scattering from the pentane isomers appears to be present. (C) 2016 AIP Publishing LLC. [http://dx.doi.org/10.1063/1.4942472]
\end{abstract}

\section{INTRODUCTION}

Investigations of lepton scattering with groups of structurally and chemically related molecules provide an opportunity to gain insights into the dynamics of the collisional process that is less apparent than in studies on just a single target (Bettega et al., 2010). Isomeric molecules represent one of those groups, as they share the same molecular formula but come in different structural configurations. Isomers typically possess different physico-chemical properties, unless they share the same functional groups, and these can affect their interaction with other systems and the environment in different ways (Petrucci et al., 2002). Isomer effects are well known, especially in organic chemistry, optical physics, and biology (Smith, 2010). In the field of atomic and molecular collisions, in particular, different isomers are expected to affect the fundamental interactions driving the scattering process in different ways (Bettega et al., 2010). An examination of the isomer effect in the electron and positron scattering dynamics is relevant for applications such as the determination of complex molecular structures (Kimura et al., 2000). There are several studies which back the existence of a clear isomer effect, especially at low incident energies, in the cross sections for electron scattering from isomeric molecules such as butanol (Bettega et al., 2010), the halocarbons $\mathrm{C}_{4} \mathrm{~F}_{6}$ (Szmytkowski and Kwitnewski, 2003b) and $\mathrm{C}_{2} \mathrm{H}_{2} \mathrm{Cl}_{2}$

\footnotetext{
a) Author to whom correspondence should be addressed. Electronic mail: luca.chiari@rs.tus.ac.jp.
}

(Kossoski et al., 2011), the hydrocarbons $\mathrm{C}_{3} \mathrm{H}_{4}$ (Nakano et al., 2002; Szmytkowski and Kwitnewski, 2002b; Lopes and Bettega, 2003; Makochekanwa et al., 2003; Sanchez et al., 2005; and Lopes et al., 2006), $\mathrm{C}_{3} \mathrm{H}_{6}$ (Mott and Massey, 1965; Floeder et al., 1985; Nishimura and Tawara, 1991; Winstead et al., 1992; Szmytkowski and Kwitnewski, 2002a; Makochekanwa et al., 2005; 2008; and Tan et al., 2007), $\mathrm{C}_{4} \mathrm{H}_{6}$ (Szmytkowski and Kwitnewski, 2003a and Lopes et al., 2004a), $\mathrm{C}_{4} \mathrm{H}_{8}$ (Lopes et al., 2004b and Bettega et al., 2006), and $\mathrm{C}_{4} \mathrm{H}_{10}$ (Floeder et al., 1985; Lopes et al., 2004b; and Bettega et al., 2007), as well as five-membered heterocyclic, aromatic compounds (Kossoski and Bettega, 2013). On the other hand, very little is known about the interaction of positrons with isomers, with just a few investigations for hydrocarbons (Floeder et al., 1985; Kimura et al., 2000; Makochekanwa et al., 2003; 2008; Sueoka et al., 2005; and Nunes et al., 2015) and chiral enantiomers (Chiari et al., 2012a) being available. Hence, while the isomer effect seems quite distinct in low-energy electron collisions, it is not yet apparent in positron scattering processes.

Isomers come in two main forms, constitutional (structural) or spatial isomers (stereoisomers), with many different subclasses such as enantiomers and diastereomers (Smith, 2010). The pentane molecule $\left(\mathrm{C}_{5} \mathrm{H}_{12}\right)$, an alkane with five carbon atoms, is an example of a structural isomer where the atoms may come in three different positional configurations: normal-pentane ( $n$-pentane), isopentane, and neopentane (see Fig. 1). The interesting aspect of this group of isomers is that they possess very similar values 
<smiles>CCCC</smiles><smiles>CCC(C)C</smiles><smiles>CC(C)(C)C(C)(C)C</smiles>

FIG. 1. Schematic structure of the three constitutional isomers of pentane: (from left to right) $n$-pentane, isopentane, and neopentane.

for their most significant physico-chemical properties, such as their permanent dipole moment (isopentane is slightly polar whereas $n$-pentane and neopentane are non-polar), static dipole polarizability, molecular diameter, and first ionization energy (see Table I). This might be due to the presence of the same functional groups in those three isomers despite their different structures. Given this similarity in their properties, the pentane isomers possibly represent one of the most suitable set of targets to carry out a stringent test for the isomer effect in lepton scattering. In particular, it would be interesting to check whether the shape and magnitude of the cross sections measured by lepton impact reflect that similarity or if the dissimilar geometry of the molecules plays any role, for instance, in the angular distributions. Unfortunately, very little data exist in the literature for electron scattering from the pentanes and are typically limited to $n$-pentane only (Freeman et al., 1979; Floriano et al., 1986; Kimura et al., 2000; and Fedus et al., 2015). With respect to positron collisions, there is only one earlier study by Kimura et al. (2000) on normal-, iso-, and cyclo-pentane (note that the latter is not an isomer of pentane, despite its name). However, in that review paper, only the data for $n$-pentane were reported. Therefore, previous work on isomer effects for lepton scattering from the pentane molecules has been limited and the results inconclusive.

In order to improve the situation for positron collisions with isomeric molecules, we have conducted an extensive experimental and theoretical investigation into positron scattering from the pentane isomers. Total cross sections (TCSs) for $n$-pentane and isopentane were measured using the positron spectrometer at the University of Trento in the energy range between 0.1 and $50 \mathrm{eV}$. In order to complete the data set for the pentane isomers and compare to the experimental data,

TABLE I. Some important physico-chemical properties of the pentane isomers: permanent dipole moment $(\mu)$, static dipole polarizability $(\alpha)$, hardsphere diameter $(D)$, first ionization energy (IP), and Ps formation threshold energy (Ps).

\begin{tabular}{lccccc}
\hline \hline Isomer & $\mu(\mathrm{D})$ & $\alpha$ (a.u.) & $D(\AA)$ & $\mathrm{IP}(\mathrm{eV})$ & $\operatorname{Ps}(\mathrm{eV})$ \\
\hline$n$-pentane & $0.006^{\mathrm{a}}$ & $67.42^{\mathrm{b}}$ & $5.575^{\mathrm{c}}$ & $10.35^{\mathrm{d}}$ & 3.55 \\
Isopentane & $0.13^{\mathrm{e}}$ & $68.36^{\mathrm{f}}$ & $5.609^{\mathrm{c}}$ & $10.32^{\mathrm{d}}$ & 3.52 \\
Neopentane & 0 & $68.83^{\mathrm{g}}$ & $5.501^{\mathrm{c}}$ & $10.35^{\mathrm{d}}$ & 3.55 \\
\hline \hline
\end{tabular}

\footnotetext{
${ }^{\mathrm{a}}$ Lewis (1989).

${ }^{\mathrm{b}}$ Maryott and Buckley (1953).

${ }^{\mathrm{c}}$ Ben-Amotz and Herschbach (1990).

${ }^{\mathrm{d}}$ Watanabe et al. (1962).

e Mopsik (1969).

${ }^{\mathrm{f}}$ Bosque and Sales (2002).

$\mathrm{g}_{\text {Applequist et al. (1972). }}$
}

calculations of the TCSs, the elastic integral cross sections (ICSs) and differential cross sections (DCSs), the positronium (Ps) formation cross sections, and the ICSs for the electronic excitations plus direct ionization were also performed for all three molecules using the independent atom model with screening corrected additivity rule (IAM-SCAR) at impact energies from 1 to $1000 \mathrm{eV}$.

The remainder of this paper is organized as follows. In Section II, we outline the present experimental techniques and in Section III, our theoretical method and computational procedures are described. Our results are then presented and discussed in Section IV. Finally, in Section V, we draw some conclusions from the findings of the present study.

\section{EXPERIMENTAL DETAILS}

The TCSs for positron scattering from $n$-pentane and isopentane were measured using the now decommissioned positron spectrometer at the University of Trento. That apparatus, as well as the experimental procedures and data analysis techniques, was comprehensively described in our earlier studies (see, e.g., Zecca et al., 2011a). Therefore we do not repeat those details here and just briefly summarize the main aspects of the experiment. A low-energy positron beam is generated by a radioactive ${ }^{22} \mathrm{Na}$ source (activity $\sim 1.3$ $\mathrm{mCi}$ ) in conjunction with a $1 \mu \mathrm{m}$-thick W moderator (Zecca et al., 2010). The positrons are transported and focused using electrostatic optics and a weak axial magnetic field $(B \approx 12 \mathrm{G})$ into the scattering cell where they interact with the target molecules. The transmitted positrons are then detected using a channel electron multiplier.

The TCSs are determined using the Beer-Lambert law (see Equation (1) in the work of Zecca et al. (2011a)) from measurements of the transmitted positron intensity and the pressure in the scattering chamber in the presence and absence of the target vapor. Knowledge of the length of the interaction region and measurements of the temperature of the target are also required. The TCSs are corrected for some instrumental effects that inevitably affect the measurements, such as the thermal transpiration effect $(<3 \%)$ and the positron effective path length increase $(<6 \%)$ due to their gyration in the applied magnetic field. Double scattering events are minimized by controlling the target pressure such that the transmittance of the beam is kept above 0.7. Prior to beginning new measurements on each target, preliminary data were collected using reference targets, such as the noble gases (Zecca et al., 2011b; 2012a; 2012b; and Chiari and Zecca, 2014) or molecular nitrogen (Zecca et al., 2011a), 
to confirm the validity of our experimental procedures. The present measurements used high-purity $n$-pentane (>99\%) and isopentane $(>99.5 \%)$ purchased from Sigma-Aldrich.

The energy distribution and the energy zero of the positron beam are determined through a retarding potential analysis (RPA) of the incident beam, without the target vapor in the chamber. The RPA measurements show that the energy resolution of the beam is $\sim 0.25 \mathrm{eV}$ (full width at half maximum, FWHM) and allow us to determine the zero of the energy scale to within $\pm 0.05 \mathrm{eV}$. The measured TCSs are convoluted over the energy distribution of the positron beam. However, this effect is only expected to be important at incident energies below $\sim 0.5 \mathrm{eV}$.

The TCSs measured at Trento are uncorrected for the forward angle scattering effect (Sanz et al., 2013a). Therefore, they represent a lower bound on the "true" TCS values. The extent of the forward scattering correction can be estimated if the angular discrimination of the spectrometer as a function of the incident energy is known and the elastic DCSs for the target of interest are available. This effect is anticipated to be more significant for polar molecules, such as isopentane (see Table I), given the more forward-peaked DCSs of those targets. Whilst the missing angle in the Trento spectrometer can be estimated at different impact energies (Zecca et al., 2011a), elastic DCSs can be calculated, for instance, using our theoretical approach (see Section III). Using those IAM-SCAR elastic DCSs for pentane, and elastic DCSs averaged over the rotational excitations for isopentane, we corrected our measured TCSs for $n$-pentane and isopentane at a few selected impact energies. The forward scattering correction is smaller than $6 \%$ for pentane (Fig. 2) and $<7 \%$ for isopentane (Fig. 3) between 1 and $50 \mathrm{eV}$. However, given the lack of experimental DCSs or independently computed DCSs for pentane and isopentane, against which we might validate our calculations, we have not documented our estimates for the forward scattering correction at all incident energies.

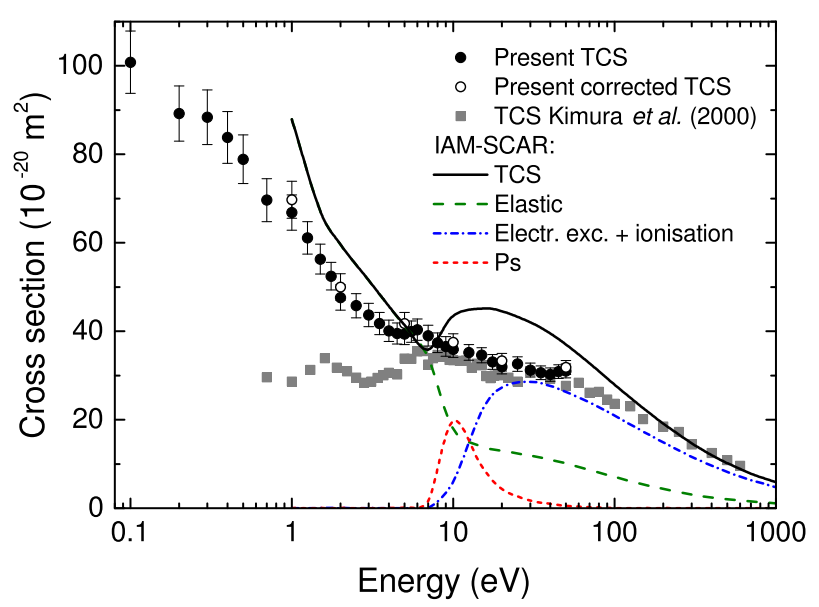

FIG. 2. Present experimental TCSs for positron scattering from $n$-pentane. The TCSs corrected for the forward angle scattering effect (see text) are given at selected incident energies. The IAM-SCAR calculations of the TCS, the elastic ICS, the Ps formation cross section, and the ICS for electronic excitations plus direct ionization are also shown. In addition, the experimental TCSs of Kimura et al. (2000) are plotted. See the legend in the figure for details.

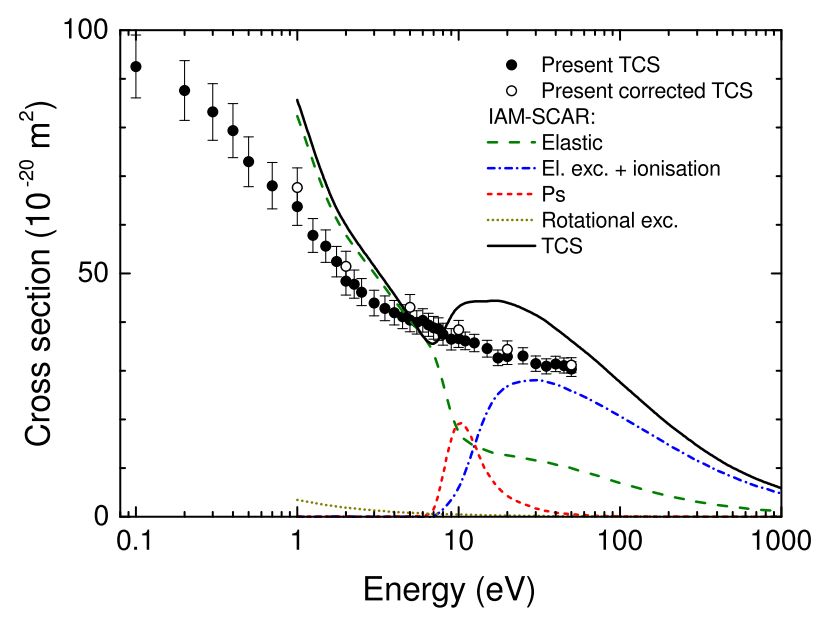

FIG. 3. The present measured TCSs for positron collisions with isopentane are shown together with the corresponding values corrected for the forward angle scattering effect (see text) at selected incident energies. The TCS, the elastic ICS, the Ps formation cross section, the ICSs for electronic excitations plus direct ionization and the rotational excitations computed using the IAM-SCAR method are also shown. See the legend in the figure for details.

Both TCS measurements on $n$-pentane and isopentane cover the positron impact energy range from 0.1 to $50 \mathrm{eV}$. The statistical uncertainties on the TCS values for $n$-pentane are typically $<4 \%$, whereas for isopentane they are $<5 \%$. Systematic sources of uncertainty in the experiments include the errors in the pressure and temperature measurements $(<1 \%$ each), in the approach used for the thermal transpiration correction $(<3 \%)$ and in the length of the scattering region and its correction for the effective positron path length $(<3.5 \%)$. Hence, the overall errors on the TCSs are estimated to range between $5 \%$ and $7 \%$ for both $n$-pentane and isopentane.

\section{THEORY AND COMPUTATIONAL DETAILS}

The IAM-SCAR method was developed by Blanco and García (2009) and has been recently applied to numerous calculations of electron (see, e.g., Chiari et al., 2013a; 2014a; and Sieradzka et al., 2014) and positron (e.g., Sanz et al., 2013b; Anderson et al., 2014; and Chiari et al., 2015) scattering cross sections from a variety of large polyatomic molecules and over an extensive impact energy range, typically from 1 to $1000 \mathrm{eV}$. Therefore, here we only briefly summarize the prominent aspects of this approach. The interested reader may refer to our previous work (Chiari et al., 2012b) for a complete description of the individual components of the scattering potential employed in the present calculations. Our formalism is based on an atomic optical potential model for the individual atoms of the target molecule, that is, hydrogen and carbon. The local complex potential is given by

$$
V(r)=V_{\mathrm{s}}(r)+V_{\mathrm{p}}(r)+\mathrm{i} V_{\mathrm{a}}(r) .
$$

The real part of Eq. (1) drives the elastic scattering process and includes $V_{\mathrm{s}}(r)$, the electrostatic potential, and $V_{\mathrm{p}}(r)$, the polarization potential. The imaginary part $V_{\mathrm{a}}(r)$ represents the inelastic processes that are considered as absorptions of flux from the incident positron beam. The static potential stems from the charge density obtained from Hartree-Fock atomic 
wavefunctions, using a similar procedure to that employed by Reid and Wadehra (1994; 1996; 1997). The dipole plus quadrupole polarization potential was developed from that reported by McEachran et al. (1977), while the absorption potential accounts for the electronic excitations, Ps formation, and direct ionization. The Ps formation channel is modelled here using the phenomenological approach adopted by Chiari et al. (2012b).

The additivity rule (AR) is then applied to the optical model results for each constituent atom in order to calculate the cross sections for positron scattering from the pentane molecules. In this approach, the molecular scattering amplitude stems from the sum of all the relevant atomic amplitudes, including the phase coefficients, which gives the DCSs for the molecule of interest. ICSs can then be determined by integrating those DCSs, with the sum of the elastic and absorption ICSs (for all inelastic processes except rotations and vibrations) then giving the TCS. The geometry of the molecule (atomic positions and bond lengths) is taken into account by using some screening coefficients which possibly extend the validity of this model down to impact energies of $30 \mathrm{eV}$ or lower.

The IAM-SCAR approach described above does not account for vibrational and rotational excitations. However, for polar molecules such as isopentane (see Table I), additional dipole-induced excitation cross sections can be calculated in the framework of the first Born approximation. These results can then be incorporated into our IAM-SCAR calculation in an incoherent way, just by adding up the cross sections as independent channels. The complete approach has already been described in detail (Chiari et al., 2014b) and proved to be quite successful when applied to some polar molecules (see, e.g., Sanz et al., 2013b and Chiari et al., 2013b).

\section{RESULTS AND DISCUSSION}

\section{A. n-pentane}

We show in Fig. 2 the present measured TCSs for positron scattering from $n$-pentane. The corresponding numerical values are reported in Table II. We see that the TCS decreases monotonically in magnitude as a function of the incident energy except for a small bump centered at around $6 \mathrm{eV}$, which is likely to arise from the opening of the Ps formation channel (see Table I). This TCS behavior is not surprising as we have observed similar trends in our earlier investigations on positron collisions with non-polar polyatomic molecules (e.g., Bettega et al., 2012; Chiari et al., 2013c; and 2014c). In those studies, we mainly ascribed that kind of behavior in the TCS to the attractive dipole interaction between the incident positron and the target molecule, which can overcome the Coulomb repulsion at those low impact energies. However, we note that the presence of a virtual state for positron binding at very low energies was also found to contribute to the sharp increase in the TCS for some of those targets (Bettega et al., 2012; Zecca et al., 2012c; and Chiari et al., 2013c). The very low-energy behaviour of the TCSs plotted in Fig. 2 seems to suggest the presence of a virtual state for the positron- $n$ pentane scattering system as well. Unfortunately, the range of
TABLE II. Present measured TCSs for positron scattering from $n$-pentane. The errors represent the overall uncertainties on the TCSs.

\begin{tabular}{lcc}
\hline \hline Energy $(\mathrm{eV})$ & TCS $\left(10^{-20} \mathrm{~m}^{2}\right)$ & TCS error $\left(10^{-20} \mathrm{~m}^{2}\right)$ \\
\hline 0.10 & 100.83 & 7.06 \\
0.20 & 89.22 & 6.25 \\
0.30 & 88.38 & 6.19 \\
0.40 & 83.81 & 5.87 \\
0.50 & 78.89 & 5.52 \\
0.70 & 69.64 & 4.87 \\
1.00 & 66.83 & 4.01 \\
1.25 & 61.14 & 3.67 \\
1.50 & 56.31 & 3.38 \\
1.75 & 52.46 & 3.15 \\
2.00 & 47.62 & 2.86 \\
2.50 & 45.77 & 2.75 \\
3.00 & 43.68 & 2.62 \\
3.50 & 41.73 & 2.50 \\
4.00 & 40.08 & 2.40 \\
4.50 & 39.48 & 2.37 \\
5.00 & 39.36 & 2.36 \\
5.50 & 39.92 & 2.40 \\
6.00 & 40.35 & 2.42 \\
7.00 & 39.00 & 2.34 \\
8.00 & 37.41 & 2.24 \\
9.00 & 36.57 & 2.19 \\
10.00 & 35.89 & 1.79 \\
12.50 & 35.21 & 1.76 \\
15.00 & 34.58 & 1.73 \\
17.50 & 33.05 & 1.65 \\
20.00 & 31.97 & 1.60 \\
25.00 & 32.58 & 1.63 \\
30.00 & 31.22 & 1.56 \\
35.00 & 30.63 & 1.53 \\
40.00 & 30.21 & 1.51 \\
45.00 & 30.91 & 1.55 \\
50.00 & 31.04 & 1.55 \\
\hline \hline & & \\
\hline & & \\
\hline
\end{tabular}

validity of the present IAM-SCAR formalism does not allow us to investigate the presence of any potential virtual states in the calculated TCS (see below). Hence the aforementioned speculation cannot be ascertained. The TCSs corrected for the forward angle scattering effect (see Section II) at some selected impact energies are also plotted in Fig. 2. As the corrections are small, they have little effect on the shape of the TCS.

The IAM-SCAR calculations of the TCS, elastic ICS, Ps formation cross section, and ICS for the electronic excitations plus direct ionization for positron impact with $n$-pentane are also shown in Fig. 2. Overall the theoretical TCS displays a qualitatively similar trend to the experimental data; however its magnitude is generally larger except between 5 and $8 \mathrm{eV}$. Below $\sim 5 \mathrm{eV}$ the discrepancy between theory and experiment might be explained by an insufficiently low forward scattering correction to the measured TCSs and/or by the IAM-SCAR method not being fully valid at those very low incident energies. At energies above $\sim 10 \mathrm{eV}$, we observe that the maximum in the IAM-SCAR ICS for the electronic excitations plus direct ionization has almost the same magnitude as the measured TCS. This is clearly unphysical given that other 
channels, such as elastic scattering and Ps formation, are also open at those energies. Hence, we believe that the IAM-SCAR calculation might be somewhat overestimated at energies just above $\sim 10 \mathrm{eV}$. We also note that the onset of the IAM-SCAR Ps formation cross section occurs at a little higher energy than the known experimental value (Table I). A similar observation was made in our earlier investigations (e.g., Chiari et al., 2014c and 2015) and was attributed to a limitation in our phenomenological approach to Ps formation. This effect probably also contributes to the overestimation of the IAM-SCAR TCS at energies above $\sim 8 \mathrm{eV}$.

In Fig. 2, we additionally plot the only previous TCS for $n$-pentane as measured by Kimura et al. (2000). That TCS shows a very good level of accord with the present experimental data (to within the combined overall uncertainties) at incident energies between about $6 \mathrm{eV}$ and the highest energy measured in Trento. Below that energy range the two data sets diverge in shape and magnitude, most likely because of a larger forward angle scattering effect affecting the earlier measurements of the Japanese group at those low energies. As discussed in detail by Hamada and Sueoka (1994), that effect for the Yamaguchi University spectrometer is the largest at very low energies and becomes less significant as the impact energy is increased. It is, therefore, not surprising to see in Fig. 2 that at higher energies $(>100 \mathrm{eV})$ the present IAM-SCAR TCS is in excellent agreement with the data of Kimura et al. (2000). This observation also likely confirms the validity of the present theoretical formalism at those incident energies.

\section{B. Isopentane}

We present in Fig. 3 our experimental TCSs for positron scattering from isopentane and give the corresponding list of tabulated values in Table III. We find a very similar picture to that just described for $n$-pentane (see Fig. 2), i.e., a monotonically decreasing TCS as the energy is increased with a slight hump at around $6 \mathrm{eV}$. However, in this case, the small permanent dipole moment of isopentane might also contribute to the overall attractive interaction between the incident positron and the target molecule, which is responsible for the shape of the low-energy TCS. We also show in Fig. 3 the measured TCSs after correction for the forward scattering effect (see Section II) at a few selected energies. That correction is again not significant here and slightly changes the shape of the TCS at the lowest energies only.

The IAM-SCAR results for the TCS, elastic ICS, Ps formation cross section, ICSs for the electronic excitations plus direct ionization and the rotational excitations for isopentane are also shown in Fig. 3. The overall qualitative behavior of this TCS is similar to that of the experimental data, i.e., it decreases in magnitude as a function of the energy with a brief increase in the cross section around the opening of the inelastic channels. In addition, the comparison of the IAM-SCAR and measured TCSs for isopentane shows a very similar picture to that seen in Fig. 2 for $n$-pentane. Hence the differences observed between theory and experiment in Fig. 3 can be explained in an analogous manner.
TABLE III. Present experimental TCSs for positron collisions with isopentane. The errors represent the overall uncertainties on the TCSs.

\begin{tabular}{|c|c|c|}
\hline Energy $(\mathrm{eV})$ & $\operatorname{TCS}\left(10^{-20} \mathrm{~m}^{2}\right)$ & TCS error $\left(10^{-20} \mathrm{~m}^{2}\right)$ \\
\hline 0.10 & 92.54 & 6.48 \\
\hline 0.20 & 87.62 & 6.13 \\
\hline 0.30 & 83.19 & 5.82 \\
\hline 0.40 & 79.34 & 5.55 \\
\hline 0.50 & 72.96 & 5.11 \\
\hline 0.70 & 68.04 & 4.76 \\
\hline 1.00 & 63.74 & 3.82 \\
\hline 1.25 & 57.82 & 3.47 \\
\hline 1.50 & 55.63 & 3.34 \\
\hline 1.75 & 52.43 & 3.15 \\
\hline 2.00 & 48.45 & 2.91 \\
\hline 2.25 & 47.81 & 2.87 \\
\hline 2.50 & 46.15 & 2.77 \\
\hline 3.00 & 43.91 & 2.63 \\
\hline 3.50 & 42.83 & 2.57 \\
\hline 4.00 & 41.93 & 2.52 \\
\hline 4.50 & 41.12 & 2.47 \\
\hline 5.00 & 40.43 & 2.43 \\
\hline 5.50 & 39.92 & 2.40 \\
\hline 6.00 & 40.36 & 2.42 \\
\hline 6.50 & 39.42 & 2.37 \\
\hline 7.00 & 38.83 & 2.33 \\
\hline 7.50 & 38.57 & 2.31 \\
\hline 8.00 & 37.51 & 2.25 \\
\hline 9.00 & 36.47 & 2.19 \\
\hline 10.00 & 36.60 & 1.83 \\
\hline 11.00 & 36.13 & 1.81 \\
\hline 12.50 & 35.72 & 1.79 \\
\hline 15.00 & 34.55 & 1.73 \\
\hline 17.50 & 32.66 & 1.63 \\
\hline 20.00 & 32.93 & 1.65 \\
\hline 25.00 & 33.06 & 1.65 \\
\hline 30.00 & 31.44 & 1.57 \\
\hline 35.00 & 30.92 & 1.55 \\
\hline 40.00 & 31.40 & 1.57 \\
\hline 45.00 & 31.07 & 1.55 \\
\hline 50.00 & 30.37 & 1.52 \\
\hline
\end{tabular}

\section{Neopentane}

Figure 4 shows the present IAM-SCAR computations of the TCS, elastic ICS, Ps formation cross section, and inelastic ICS (accounting again for the electronic excitations and direct ionization) for positron collisions with neopentane. The behavior of all those cross sections is very similar to that of the corresponding IAM-SCAR cross sections for $n$-pentane and isopentane (see Figs. 2 and 3). That is, the TCS again displays a monotonic decrease in magnitude as a function of the energy, up to the opening of the first inelastic channel, then rises up to a relative maximum at $\sim 20 \mathrm{eV}$ before falling in magnitude again. Similar to n-pentane (see Section IV A), the presence of a virtual state for very low-energy positron binding to neopentane might also contribute to the TCS behaviour observed in Fig. 4. However, as explained in Section IV A, the nature of our IAM-SCAR approach does not enable us to check the presence of such a virtual state in the calculations. 


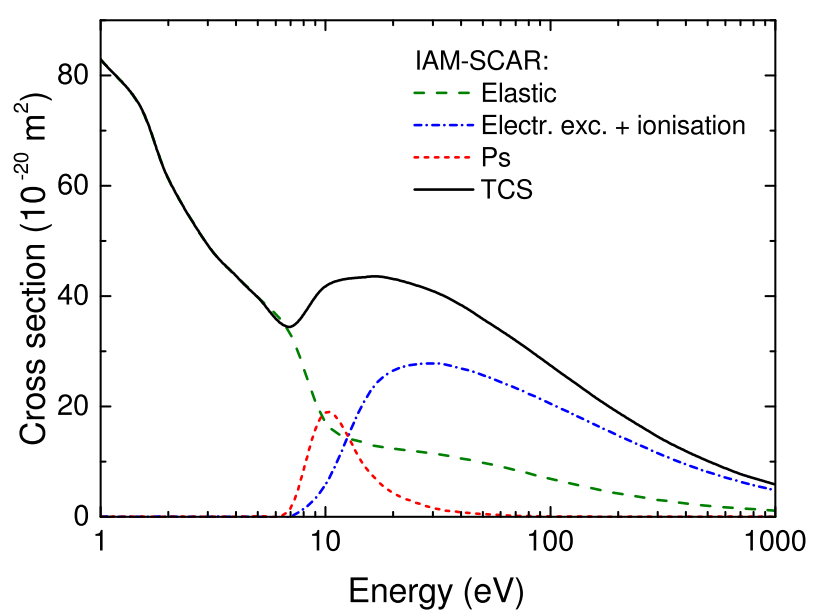

FIG. 4. Present IAM-SCAR results for positron impact with neopentane. The TCS, elastic ICS, Ps formation cross section, and the ICS for the electronic excitations plus direct ionization are shown. See the legend in the figure for details.

Unfortunately, there is no experimental data for neopentane against which we can compare our theoretical results.

\section{Comparison of the three isomers: Isomer effect}

We now compare the experimental and theoretical cross sections for the three pentane isomers in order to understand whether or not an isomer effect is present in the cross sections for those targets. As the most significant physico-chemical properties of the pentane isomers are very similar to each other (see Table I), it might be reasonable to ascribe any significant discrepancies in their cross sections (if any) to their different geometrical structures playing a major role in the scattering dynamics.

In Fig. 5, we compare the present experimental and theoretical TCSs for the three pentane isomers. The measured TCSs for $n$-pentane and isopentane appear to be consistent with each other to within the uncertainties on the data at all incident energies. The TCSs for the three isomers computed

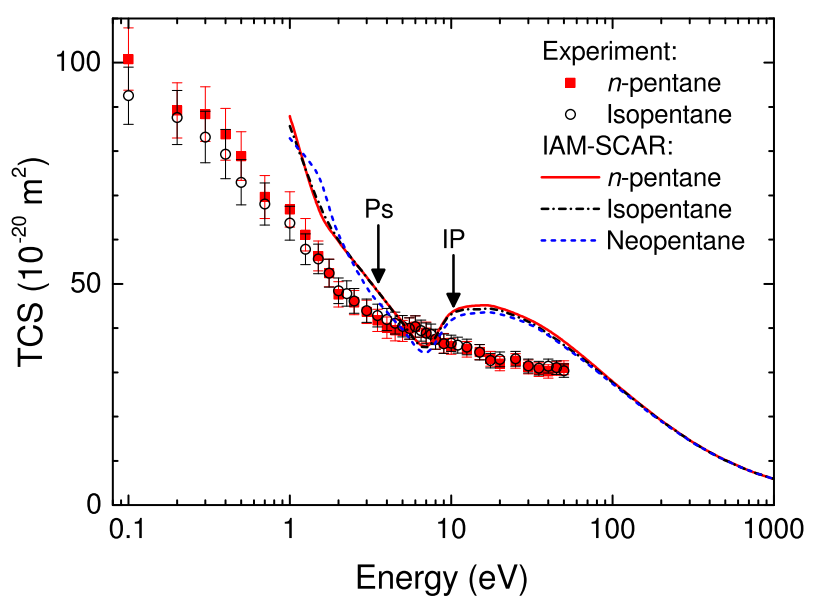

FIG. 5. Comparison of the present TCSs for positron scattering from the three pentane isomers. The experimental TCSs for $n$-pentane and isopentane are shown together with the IAM-SCAR TCSs for each of the three isomers. See the legend in the figure for details. using the IAM-SCAR method overlap with each other except at the lower energies. Specifically, below $\sim 5 \mathrm{eV}$, the TCS for neopentane appears to slightly deviate from the results of the other two calculations. However, given that we do not expect the IAM-SCAR approach to be fully valid at those low incident energies, the aforementioned discrepancy might as well be considered to be within the reliability on those calculations and not be necessarily related to any underlying physical effect. In conclusion, we believe that no significant isomer effect seems to be apparent in the TCSs, either experimental or theoretical, for positron collisions with the pentane isomers.

A perhaps more stringent test of the presence of any isomer effects in positron scattering from these molecules may be offered by the comparison of their Ps formation cross sections, or their angular distributions for elastic scattering. The Ps formation cross sections for the three pentane isomers calculated using the IAM-SCAR method are reported in Fig. 6. Those cross sections are almost identical to each other in both shape and magnitude. Given the semi-empirical approach we use to incorporate Ps formation into the model, this result is possibly inherent to the way the computation is set up. An exception to the good agreement observed for the three molecules might be found at their peak at around $10 \mathrm{eV}$, where the cross section for $n$-pentane seems to be just slightly larger in magnitude than the other two cross sections. However, given the limitations of our IAM-SCAR formalism for this scattering channel, that we mentioned earlier, we believe that the aforementioned difference lies also well within the reliability of the computations.

Figure 7 shows a comparison of the elastic DCSs for the three pentane isomers, calculated using the IAM-SCAR approach, at six selected impact energies between 1 and $50 \mathrm{eV}$. All the DCSs show a forward-peaked shape which is typical of polar molecules (see, e.g., Tattersall et al., 2014; Chiari et al., 2013a, 2013d; and Palihawadana et al., 2013) and become more isotropic as the incident positron energy is decreased. Note that the DCSs for isopentane are averaged over the rotational excitations, whereas those for the other

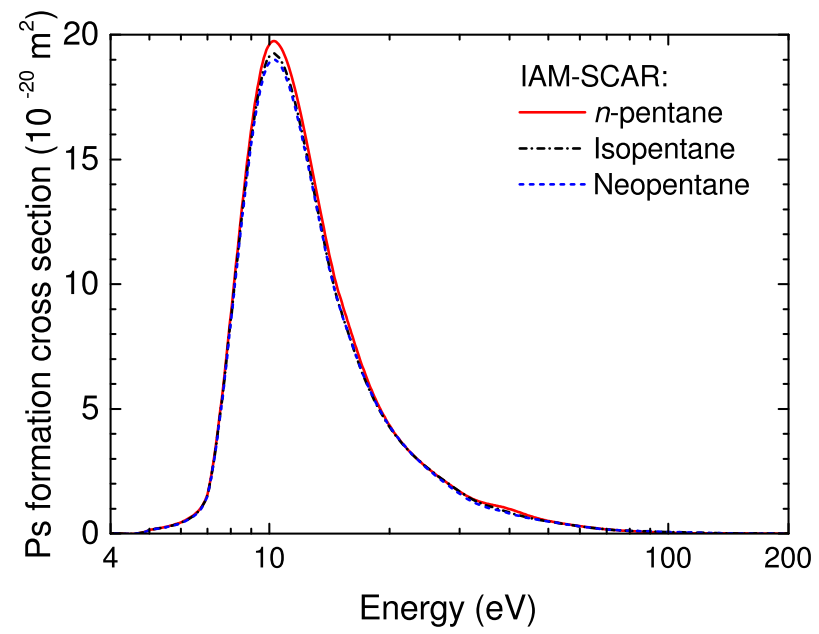

FIG. 6. Comparison of the present Ps formation cross sections for positron scattering from the pentane isomers calculated with our IAM-SCAR approach. See the legend in the figure for details. 


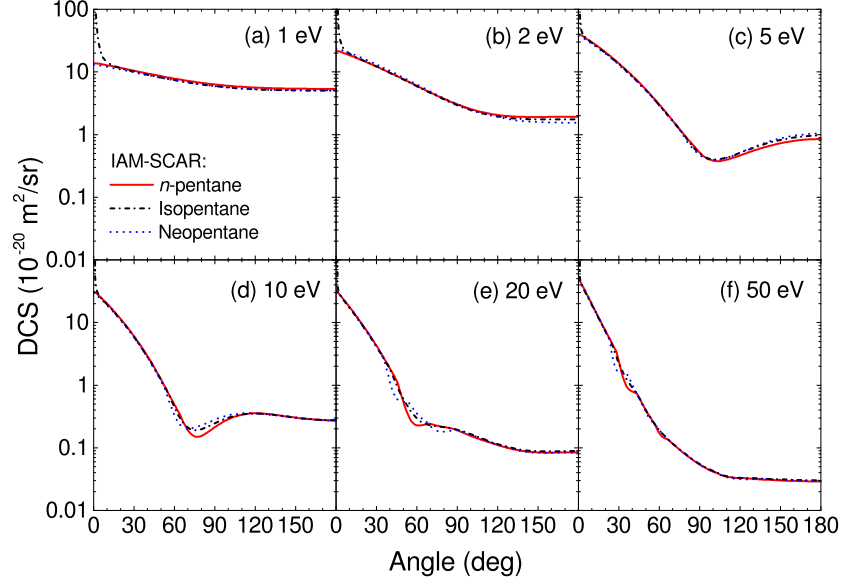

FIG. 7. The present elastic DCSs for positron scattering from the pentane isomers computed using the IAM-SCAR formalism are shown at selected incident energies: (a) 1, (b) 2, (c) 5, (d) 10, (e) 20, and (f) $50 \mathrm{eV}$. Note that the elastic DCSs for isopentane are averaged over the rotational excitations. See the legend in the figure for details.

two isomers are not given that they are non-polar. For this reason the DCSs for isopentane become very forward peaked below $\sim 10^{\circ}$ at all energies, unlike those for $n$-pentane and neopentane. Except in this very forward angular range, the DCSs for the three isomers basically overlap in value with each other at incident energies up to $5 \mathrm{eV}$. However, at energies greater than $10 \mathrm{eV}$, some slight differences in the shape and magnitude of the three DCSs become apparent at the intermediate angles. It also seems that those differences become somewhat larger as the impact energy is increased (note the cross section axis log-scale). Remembering our caveat about the validity of the IAM-SCAR method at those low incident energies, it is unclear whether those differences in the DCSs can be definitively attributed to an isomer effect or not. At $10 \mathrm{eV}$, they are most likely to be within the confidence bound on the calculations. However, at 20 and $50 \mathrm{eV}$, the differences between the DCS for neopentane and those for $n$-pentane and isopentane might originate, at least in part, from the different structures of the molecules. In fact, whereas neopentane possesses spherical-like symmetry, the other two isomers do not (see Fig. 1). Although this possible isomer effect is found to be not so large here, it does appear to be non-negligible.

Consistent with the view of Kimura et al. (2000), the reason for a weak isomer effect here might be due to the relatively large size of the pentane molecular systems, so that there are many electrons and bonds that can effectively "smear out" the effect. The lack of an isomer effect for the pentane molecules might also be traced back to the absence of a bond effect, i.e., the nature of the $\mathrm{C}-\mathrm{C}$ bonding. In electron collisions with saturated ( $\mathrm{C}-\mathrm{C}$ single bonds only) versus unsaturated (double or triple bonds) isomers, it is known that the presence of one or more double or triple bonds results in the appearance of low-energy $\pi^{*}$ shape resonances in the unsaturated isomers (Makochekanwa et al., 2005). Given that all the three pentane isomers are saturated compounds (see Fig. 1), one can therefore expect no bond effect in the lepton scattering from those targets. This is exactly what we find here. Furthermore, it has been shown that the angular distributions for electron scattering from branched versus straight chain isomers are affected by the nature of the chain system (Bettega et al., 2010). Specifically, the DCSs show a different scattering behaviour ( $d$-wave versus $f$-wave) around the region of the broad resonance, depending on the type of molecular chain (branched versus straight, respectively). It is, therefore, intriguing to note that the present elastic DCSs for positron collisions with the three pentane isomers are very similar to each other at all energies, despite the fact that $n$-pentane is a straight chain system whereas isopentane and neopentane are branched chain systems.

\section{E. Comparison of the homologous series of molecules $\mathrm{C}_{n} \mathrm{H}_{2 n+2}$}

Finally, we compare in Fig. 8(a) the TCSs measured at the University of Trento for $n$-pentane (present data), ethane $\left(\mathrm{C}_{2} \mathrm{H}_{6}\right.$; Chiari et al., 2013c), and methane $\left(\mathrm{CH}_{4}\right.$; Zecca et al., 2012c). Note that the overall uncertainties on the TCSs are plotted in that figure. In Fig. 8(a), we see that the very low-energy behaviour of the TCSs for methane and ethane is very similar but different from that of the TCS for $n$-pentane. This different trend might be explained by the presence of
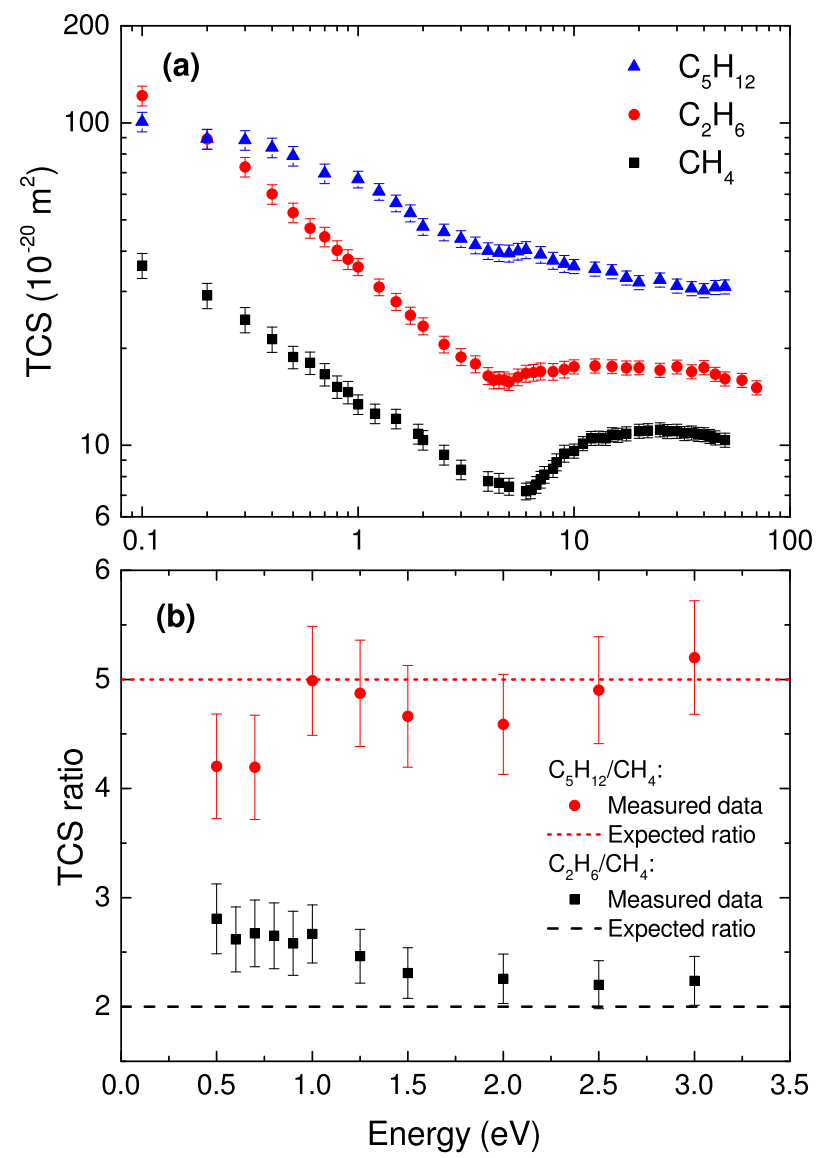

FIG. 8. (a) Comparison of the TCSs measured in Trento for positron scattering from the homologous series of molecules $\mathrm{C}_{n} \mathrm{H}_{2 n+2}$ : methane $\left(\mathrm{CH}_{4}\right.$; Zecca et al., 2012c), ethane $\left(\mathrm{C}_{2} \mathrm{H}_{6}\right.$; Chiari et al., 2013c), and $n$-pentane $\left(\mathrm{C}_{5} \mathrm{H}_{12}\right.$; present data). The overall uncertainties on the TCSs are given here. (b) Ratios of the measured TCSs for $n$-pentane and ethane to that of methane together with the expected ratios. 
a weaker virtual state, if any, in the TCS for $n$-pentane (see Section IV A) compared to methane and ethane. The rationale for this comparison is that all those molecules are part of the homologous series of compounds with the formula $\mathrm{C}_{n} \mathrm{H}_{2 n+2}$, where $n=1$ for methane, $n=2$ for ethane, and $n=5$ for $n$ pentane. Hence, it might be interesting, for instance, to check whether or not their respective TCSs scale with the number $n$ of C-atoms. However, this comparison is only meaningful for energies where the convolution with the energy distribution of the positron beam and the forward angle scattering effect are not significant, as they might be a little different for each target. In addition, the opening of the Ps formation channel might also somewhat complicate this evaluation. Therefore, we plot in Fig. 8(b) the ratios of the measured TCSs for $n$-pentane to methane and ethane to methane at incident energies between 0.5 and $3 \mathrm{eV}$ only. Dashed lines which indicate the expected TCS ratios ( 2 for ethane/methane and 5 for $n$-pentane/methane), if there is a scaling dependence on $n$, are also shown in that figure. We see in Fig. 8(b) that the ratio of the measured TCSs for ethane to methane is somewhat larger than the anticipated value of 2 at all impact energies. On the other hand, the ratios of the measured TCSs for $n$-pentane to methane are typically lower than the scaled value of 5 at all incident energies but $3 \mathrm{eV}$. However, to within the measurement uncertainties, they are all consistent with that scaled value between 1 and $3 \mathrm{eV}$. These results overall seem to indicate that the TCSs for the homologous compounds $\mathrm{C}_{n} \mathrm{H}_{2 n+2}$ do not exactly scale with $n$ and that some other molecular effects must play a role in the positron scattering process with those targets. This result is entirely consistent with work from Sophia University (Hoshino et al., 2013 and Kato et al., 2012), which clearly demonstrated the importance of molecular effects in electron-molecule scattering.

\section{CONCLUSIONS}

We have reported on cross section measurements and calculations for positron scattering from the pentane isomers. TCSs for $n$-pentane and isopentane were measured at the University of Trento in the energy range from 0.1 to $50 \mathrm{eV}$. In addition, calculations of the TCSs, elastic ICSs, Ps formation cross sections, ICSs for the electronic excitations plus direct ionization and elastic DCSs for all the pentane isomers were carried out using the IAM-SCAR approach at impact energies from 1 to $1000 \mathrm{eV}$. The measured TCSs for $n$-pentane and isopentane are consistent with each other to within the respective overall uncertainties. The TCS measurements and the IAM-SCAR calculations for each isomer exhibited a level of accord that was often limited to their shape or in magnitude to some impact energies only. The comparison between the measurements and the computations for the three pentane isomers seems to suggest the absence of any significant isomer effect, except maybe at the elastic DCS level. Nevertheless, at this time whether or not there is a clear isomer effect present, in the cross sections for positron scattering from the pentanes, remains something of an open question requiring further investigations. Given that our IAM-SCAR formalism may not be so accurate at incident energies below $30 \mathrm{eV}$ or so, $a b$ initio positron-molecule calculations, such as those based on density functional theory (DFT) or self-consistent field with configuration interaction (SCF-CI), might be more precise at those very low energies. In particular, we acknowledge the recent fundamental improvements in the electron-positron scattering potential within the DFT framework, both in the local density approximation (LDA) (Drummond et al., 2011) and the generalized gradient approximation (GGA) (Barbiellini and Kuriplach, 2015) methods. Although these theoretical approaches do not explicitly account for Ps formation, which is an important scattering channel at such low energies, they might lead to a clearer picture of the isomer effect.

\section{ACKNOWLEDGMENTS}

The experimental work at the University of Trento was undertaken under a Memorandum of Understanding with the Flinders University node of the former Australian Research Council's Centre of Excellence for AntimatterMatter Studies. G.G. and F.B. would like to acknowledge the Spanish Ministerio de Economía y Productividad (Project No. FIS2012-31230) and the European Science Foundation (COST Action Grants Nos. MP1002-Nano-IBCT and MC1301-CELINA) for financial support. Finally, L.C. thanks the Japan Society for the Promotion of Science for his fellowship.

Anderson, E. K., Boadle, R. A., Machacek, J. R., Chiari, L., Makochekanwa, C., Buckman, S. J., Brunger, M. J., Garcia, G., Blanco, F., Ingolfsson, O., and Sullivan, J. P., "Low energy positron interactions with uracil-Total scattering, positronium formation, and differential elastic scattering cross sections," J. Chem. Phys. 141, 034306 (2014).

Applequist, J., Carl, J. R., and Fung, K.-K., "An atom dipole interaction model for molecular polarizability. Application to polyatomic molecules and determination of atom polarizabilities," J. Am. Chem. Soc. 94, 2952-2960 (1972).

Barbiellini, B. and Kuriplach, J., "Proposed parameter-free model for interpreting the measured positron annihilation spectra of materials using a generalized gradient approximation,” Phys. Rev. Lett. 114, 147401 (2015).

Ben-Amotz, D. and Herschbach, D. R., "Estimation of effective diameters for molecular fluids," J. Phys. Chem. 94, 1038-1047 (1990).

Bettega, M. H. F., Lopes, A. R., Lima, M. A. P., and Ferreira, L. G., "Electron collisons with cyclobutane," Braz. J. Phys. 36, 570-575 (2006).

Bettega, M. H. F., Lima, M. A. P., and Ferreira, L. G., "Scattering of low-energy electrons by isomers of $\mathrm{C}_{4} \mathrm{H}_{10}$," J. Phys. B 40, 3015-3023 (2007).

Bettega, M. H. F., Winstead, C., and McKoy, V., "Low-energy electron scattering from $\mathrm{C}_{4} \mathrm{H}_{9} \mathrm{OH}$ isomers," Phys. Rev. A 82, 062709 (2010).

Bettega, M. H. F., Sanchez, S. d'A., Varella, M. T. do N., Lima, M. A. P., Chiari, L., Zecca, A., Trainotti, E., and Brunger, M. J., "Positron collisions with ethene," Phys. Rev. A 86, 022709 (2012).

Blanco, F. and García, G., "A screening-corrected additivity rule for the calculation of electron scattering from macro-molecules," J. Phys. B 42, 145203 (2009).

Bosque, R. and Sales, J., "Polarizabilities of solvents from the chemical composition,” J. Chem. Inf. Comput. Sci. 42, 1154-1163 (2002).

Chiari, L., Zecca, A., Girardi, S., Defant, A., Wang, F., Ma, X. G., Perkins, M. V., and Brunger, M. J., "Positron scattering from chiral enantiomers," Phys. Rev. A 85, 052711 (2012a).

Chiari, L., Zecca, A., Girardi, S., Trainotti, E., García, G., Blanco, F., McEachran, R. P., and Brunger, M. J., "Positron scattering from $\mathrm{O}_{2}$," J. Phys. B 45, 215206 (2012b).

Chiari, L., Anderson, E., Tattersall, W., Machacek, J. R., Palihawadana, P., Makochekanwa, C., Sullivan, J. P., García, G., Blanco, F., McEachran, R. P., Brunger, M. J., and Buckman, S. J., "Total, elastic and inelastic cross sections for positron and electron collisions with tetrahydrofuran," J. Chem. Phys. 138, 074301 (2013a). 
Chiari, L., Zecca, A., García, G., Blanco, F., and Brunger, M. J., "Low-energy positron and electron scattering from nitrogen dioxide," J. Phys. B 46, 235202 (2013b).

Chiari, L., Zecca, A., Trainotti, E., Bettega, M. H. F., Sanchez, S. d'A., Varella, M. T. do N., Lima, M. A. P., and Brunger, M. J., "Cross sections for positron scattering from ethane," Phys. Rev. A 87, 032707 (2013c).

Chiari, L., Palihawadana, P., Machacek, J. R., Makochekanwa, C., García, G., Blanco, F., McEachran, R. P., Brunger, M. J., Buckman, S. J., and Sullivan, J. P., "Experimental and theoretical cross sections for positron collisions with 3-hydroxy-tetrahydrofuran,” J. Chem. Phys. 138, 074302 (2013d).

Chiari, L. and Zecca, A., "Recent positron-atom cross section measurements and calculations," Eur. Phys. J. D 68, 297 (2014).

Chiari, L., Duque, H. V., Jones, D. B., Thorn, P. A., Pettifer, Z., da Silva, G. B., Limão-Vieira, P., Duflot, D., Hubin-Franskin, M.-J., Delwiche, J., Blanco, F., García, G., Lopes, M. C. A., Ratnavelu, K., White, R. D., and Brunger, M. J., "Differential cross sections for intermediate-energy electron scattering from $\alpha$-tetrahydrofurfuryl alcohol: Excitation of electronic-states," J. Chem. Phys. 141, 024301 (2014a).

Chiari, L., Zecca, A., Blanco, F., García, G., and Brunger, M. J., "Positron scattering from vinyl acetate,” J. Phys. B 47, 175202 (2014b).

Chiari, L., Zecca, A., Blanco, F., García, G., Perkins, M. V., Buckman, S. J., and Brunger, M. J., "Cross sections for positron impact with 2,2,4trimethylpentane,” J. Phys. Chem. A 118, 6466-6472 (2014c).

Chiari, L., Zecca, A., Blanco, F., García, G., and Brunger, M. J., "Cross sections for positron and electron collisions with an analog of the purine nucleobases: Indole,” Phys. Rev. A 91, 012711 (2015).

Drummond, N. D., López Ríos, P., Needs, R. J., and Pickard, C. J., "Quantum Monte Carlo study of a positron in an electron gas," Phys. Rev. Lett. 107, 207402 (2011).

Fedus, K., Navarro, C., Hargreaves, L. R., Khakoo, M. A., Barbosa, A. S., and Bettega, M. H. F., "Differential elastic electron scattering by pentane," Phys. Rev. A 91, 042701 (2015).

Floeder, K., Fromme, D., Raith, W., Schwab, A., and Sinapius, G., “Total cross section measurements for positron and electron scattering on hydrocarbons between 5 and 400 eV," J. Phys. B 18, 3347-3359 (1985).

Floriano, M. A., Gee, N., and Freeman, G. R., "Electron transport in low density alkane gases: Effects of chain length and flexibility," J. Chem. Phys. 84, 6799-6807 (1986).

Freeman, G. R., György, I., and Huang, S. S.-S., "Electron scattering cross sections of gaseous pentanes and hexanes," Can. J. Chem. 57, 2626-2628 (1979).

Hamada, A. and Sueoka, O., "Total cross section measurements for positrons and electrons colliding with molecules: II. HCl," J. Phys. B 27, 5055-5064 (1994).

Hoshino, M., Horie, M., Kato, H., Blanco, F., García, G., Limão-Vieira, P., Sullivan, J. P., Brunger, M. J., and Tanaka, H., "Cross sections for elastic scattering of electrons by $\mathrm{CF}_{3} \mathrm{Cl}, \mathrm{CF}_{2} \mathrm{Cl}_{2}$, and $\mathrm{CFCl}_{3}$," J. Chem. Phys. 138, 214305 (2013).

Kato, H., Anzai, K., Ishihara, T., Hoshino, M., Blanco, F., García, G., LimãoVieira, P., Brunger, M. J., Buckman, S. J., and Tanaka, H., "A study of electron interactions with silicon tetrafluoride: Elastic scattering and vibrational excitation cross sections," J. Phys. B 45, 095204 (2012).

Kimura, M., Sueoka, O., Hamada, A., and Itikawa, Y., in Advances in Chemical Physics, edited by Prigogine I. and Rice S. A. (Wiley, 2000), Vol. 111, pp. 537-622.

Kossoski, F., Freitas, T. C., and Bettega, M. H. F., "Resonances in electron collisions with $\mathrm{C}_{2} \mathrm{H}_{2} \mathrm{Cl}_{2}$ isomers," J. Phys. B 44, 245201 (2011).

Kossoski, F. and Bettega, M. H. F., "Low-energy electron scattering from the aza-derivatives of pyrrole, furan, and thiophene," J. Chem. Phys. 138, 234311 (2013).

Lewis, D. F., "The calculation of molar polarizabilities by the CNDO/2 method: Correlation with the hydrophobic parameter, log P,' J. Comput. Chem. 10, 145-151 (1989).

Lopes, A. R. and Bettega, M. H. F., "Elastic scattering of low-energy electrons by $\mathrm{C}_{3} \mathrm{H}_{4}$ isomers," Phys. Rev. A 67, 032711 (2003).

Lopes, A. R., Lima, M. A. P., Ferreira, L. G., and Bettega, M. H. F., "Lowenergy electron collisions with $\mathrm{C}_{4} \mathrm{H}_{6}$ isomers," Phys. Rev. A 69, 014702 (2004a).

Lopes, A. R., Bettega, M. H. F., Lima, M. A. P., and Ferreira, L. G., "Electron collisions with isomers of $\mathrm{C}_{4} \mathrm{H}_{8}$ and $\mathrm{C}_{4} \mathrm{H}_{10}$," J. Phys. B 37, 997-1012 (2004b).

Lopes, A. R., Bettega, M. H. F., Varella, M. T. do N., and Lima, M. A. P., "Cross-sections for rotational excitations of $\mathrm{C}_{3} \mathrm{H}_{4}$ isomers by electron impact,” Eur. Phys. J. D 37, 385-392 (2006).
Makochekanwa, C., Kawate, H., Sueoka, O., Kimura, M., Kitajima, M., Hoshino, M., and Tanaka, H., "Total and elastic cross-sections of electron and positron scattering from $\mathrm{C}_{3} \mathrm{H}_{4}$ molecules (allene and propyne)," Chem. Phys. Lett. 368, 82-86 (2003).

Makochekanwa, C., Kato, H., Hoshino, M., Cho, H., Kimura, M., Sueoka, O., and Tanaka, $\mathrm{H}$., "Probing the isomer, fluorination and bond effects in $\mathrm{C}_{3} \mathrm{H}_{6}$, cyclo- $\mathrm{C}_{3} \mathrm{H}_{6}$ and $\mathrm{C}_{3} \mathrm{~F}_{6}$ molecules using electron impact,' Eur. Phys. J. D 35, 249-255 (2005).

Makochekanwa, C., Hoshino, M., Kato, H., Sueoka, O., Kimura, M., and Tanaka, H., "Electron and positron scattering cross sections for propene and cyclopropane," Phys. Rev. A 77, 042717 (2008).

Maryott, A. A. and Buckley, F., "Table of dielectric constants and electric dipole moments of substances in the gaseous state," in NBS Circular 537 (U. S. National Bureau of Standards, Washington, DC, 1953).

McEachran, R. P., Ryman, A. G., Stauffer, A. D., and Morgan, D. L., "Positron scattering from noble gases," J. Phys. B 10, 663-677 (1977).

Mopsik, F. I., "Dielectric properties of slightly polar organic liquids as a function of pressure, volume, and temperature," J. Chem. Phys. 50, 2559-2569 (1969).

Mott, N. F. and Massey, H. S. W., The Theory of Atomic Collisions (Oxford University Press, Oxford, 1965).

Nakano, Y., Hoshino, M., Kitajima, M., Tanaka, H., and Kimura, M., "Lowenergy electron scattering from $\mathrm{C}_{3} \mathrm{H}_{4}$ isomers: Differential cross sections for elastic scattering and vibrational excitation," Phys. Rev. A 66, 032714 (2002).

Nishimura, H. and Tawara, H., "Some aspects of total scattering cross sections of electrons for simple hydrocarbon molecules," J. Phys. B 24, L363-L366 (1991).

Nunes, F. B., Bettega, M. H. F., and Sanchez, S. d'A., "Positron collisions with $\mathrm{C}_{3} \mathrm{H}_{6}$ isomers," J. Phys. B 48, 165201 (2015).

Palihawadana, P., Boadle, R., Chiari, L., Anderson, E. K., Machacek, J. R., Brunger, M. J., Buckman, S. J., and Sullivan, J. P., "Positron scattering from pyrimidine," Phys. Rev. A 88, 012717 (2013).

Petrucci, R. H., Harwood, R. S., and Herring, F. G., General Chemistry (Prentice-Hall, 2002).

Reid, D. D. and Wadehra, J. M., "Low-energy differential scattering of electrons and positrons from noble gases," Phys. Rev. A 50, 4859-4867 (1994).

Reid, D. D. and Wadehra, J. M., "A quasifree model for the absorption effects in positron scattering by atoms," J. Phys. B 29, L127-L133 (1996).

Reid, D. D. and Wadehra, J. M., "Erratum: A quasifree model for the absorption effects in positron scattering by atoms," J. Phys. B 30, 2318 (1997).

Sanchez, S. d'A., Lopes, A. R., Bettega, M. H. F., Lima, M. A. P., and Ferreira, L. G., "Polarization effects in the elastic scattering of low-energy electrons by $\mathrm{C}_{3} \mathrm{H}_{4}$ isomers," Phys. Rev. A 71, 062702 (2005).

Sanz, A. G., Fuss, M. C., Blanco, F., Gorfinkiel, J. D., Almeida, D., Ferreira da Silva, F., Limão-Vieira, P., Brunger, M. J., and García, G., "An investigation into electron scattering from pyrazine at intermediate and high energies," J. Chem. Phys. 139, 184310 (2013a).

Sanz, A. G., Fuss, M. C., Blanco, F., Mašín, Z., Gorfinkiel, J. D., McEachran, R. P., Brunger, M. J., and García, G., "Cross-section calculations for positron scattering from pyrimidine over an energy range from 0.1 to 10000 eV,' Phys. Rev. A 88, 062704 (2013b).

Sieradzka, A., Blanco, F., Fuss, M. C., Mašín, Z., Gorfinkiel, J. D., and García, G., "Electron scattering from pyridine," J. Phys. Chem. A 118, 6657-6663 (2014).

Smith, J. G., General Organic and Biological Chemistry (The McGraw-Hill Companies, 2010).

Sueoka, O., Makochekanwa, C., Tanino, H., and Kimura, M., "Total crosssection measurements for positrons and electrons colliding with alkane molecules: Normal hexane and cyclohexane," Phys. Rev. A 72, 042705 (2005).

Szmytkowski, C. and Kwitnewski, S., "Electron scattering on $\mathrm{C}_{3} \mathrm{H}_{6}$ isomers," J. Phys. B 35, 2613-2623 (2002a).

Szmytkowski, C. and Kwitnewski, S., "Total cross sections for electron scattering with some $\mathrm{C}_{3}$ hydrocarbons," J. Phys. B 35, 3781-3790 (2002b).

Szmytkowski, C. and Kwitnewski, S., "Electron scattering from $\mathrm{C}_{4} \mathrm{H}_{6}$ and $\mathrm{C}_{4} \mathrm{~F}_{6}$ molecules," J. Phys. B 36, 2129-2138 (2003a).

Szmytkowski, C. and Kwitnewski, S., "Isomer effects on the total cross section for electron scattering from $\mathrm{C}_{4} \mathrm{~F}_{6}$ molecules," J. Phys. B 36, 4865-4873 (2003b).

Tan, X.-M., Sun, J.-F., Shi, D.-H., and Liu, Z.-J., "Total cross-sections for electron scattering of $\mathrm{C}_{3} \mathrm{H}_{6}$ isomers: A modified additivity rule approach," Z. Naturforsch. A 62, 716-720 (2007).

Tattersall, W., Chiari, L., Machacek, J. R., Anderson, E., White, R. D., Brunger, M. J., Buckman, S. J., Garcia, G., Blanco, F., and Sullivan, J. P., "Positron 
interactions with water-total elastic, total inelastic, and elastic differential cross section measurements," J. Chem. Phys. 140, 044320 (2014).

Winstead, C., Sun, Q., and McKoy, V., "Low-energy electron scattering by $\mathrm{C}_{3} \mathrm{H}_{6}$ isomers," J. Chem. Phys. 96, 4246-4251 (1992).

Watanabe, K., Nakayama, T., and Mottl, J., "Ionization potentials of some molecules,” J. Quant. Spectrosc. Radiat. Transfer 2, 369-382 (1962).

Zecca, A., Chiari, L., Sarkar, A., Chattopadhyay, S., and Brunger, M. J., "Procedures for conditioning $\mathrm{W}$ - and Ni-moderators for application in positron-scattering measurements," Nucl. Instrum. Methods Phys. Res., Sect. B 268, 533-536 (2010).

Zecca, A., Chiari, L., Sarkar, A., and Brunger, M. J., "Positron scattering from the isoelectronic molecules $\mathrm{N}_{2}, \mathrm{CO}$ and $\mathrm{C}_{2} \mathrm{H}_{2}$," New J. Phys. 13, 115001 (2011a).
Zecca, A., Chiari, L., Trainotti, E., Fursa, D. V., Bray, I., and Brunger, M. J., "Experimental determination of the scattering length for positron scattering from krypton,” Eur. Phys. J. D 64, 317-321 (2011b).

Zecca, A., Chiari, L., Trainotti, E., Fursa, D. V., Bray, I., Sarkar, A., Chattopadhyay, S., Ratnavelu, K., and Brunger, M. J., "Positron scattering from argon: Total cross sections and the scattering length,' J. Phys. B 45, 015203 (2012a).

Zecca, A., Chiari, L., Trainotti, E., and Brunger, M. J., "Very low-energy total cross sections and the experimental scattering length for the positron-xenon system," J. Phys. B 45, 085203 (2012b).

Zecca, A., Chiari, L., Trainotti, E., Sarkar, A., Sanchez, S. d'A., Bettega, M. H. F., Varella, M. T. do N., Lima, M. A. P., and Brunger, M. J., "Positron scattering from methane," Phys. Rev. A 85, 012707 (2012c). 